\title{
A Phenomenological Model For Collisional Coherence Transfer In Optically Pumped Atomic System
}

\author{
K. Khanbekyan ${ }^{1, *}$, G. Bevilaqua ${ }^{1}$, A. Khanbekyan ${ }^{2}$, E. \\ Mariotti $^{1}$, A. Papoyan ${ }^{2}$ and L. Moi ${ }^{1}$ \\ ${ }^{1}$ Università degli Studi di Siena, Siena, 53100 Italy \\ ${ }^{2}$ Institute for Physical Research, National Academy of Sciences - Ashtarak 2, Armenia \\ E-mail: * karen.khanbekyan@gmail.com
}

PACS numbers: 32.70.-n, 32.70.Jz, 33.40.+f, 33.57.+c

\begin{abstract}
We consider a dual $\Lambda$-system under double laser excitation to investigate the possibility of indirect coherence transfer between atomic ground states through an excited state. The atomic system is excited by a frequency modulated pump laser and probed by a low-power cw laser. All the decoherence mechanisms are discussed and taken into account. Adjustment of parameters of the two radiations aimed at maximization of coherence transfer is addressed. The study can help to understand phenomena as collisional transfer of coherence and can find application in the experimental realization of atomic sensors.
\end{abstract}




\section{Introduction}

Optical pumping technique serves as a powerful tool for spin polarizing an atomic sample since early fifties [Kastler(1950)], see also the review [Happer(1972)]. Modulating a laser beame is a well known issue since the pioneristic work of [Series (1966)] and can be profitable used to observe resonant effects. These experimental methods recently attracted renewed attention due to its various applications in many different fields and its potential importance in the development of atomic probes of highest sensitivity.

Optical atomic magnetometers constitute a significant example. In fact sensitivities as high as $22 \mathrm{fT} / \sqrt{\mathrm{Hz}}$ have been obtained $[\operatorname{Shah}(2010)]$, that, although are not comparable with those of SQUID sensors $\left(\approx 10^{-1} \mathrm{fT} / \sqrt{\mathrm{Hz}}\right)$, allow for the possibility of miniaturizing the sensor volume [Schwindt(2004)] [Ledbetter(2008)] or of getting rid of cryogenics as they work at room temperature or higher. Recent experiments demonstrated the possibility of low field NMR [Belfi(2009)]. In last paper a laser beam is frequency modulated to produce a pulsed excitation of atoms. This creates an atomic polarization that, due to an external magnetic field, precesses at Larmor frequency. The aim of that work was to build a new self oscillating optical magnetometer based on non linear magneto optical rotation and to open a way to new applications and ideas. For example, shining another transition of the atomic sample by using a cw probe laser we expect to find a transferred modulated behaviour on the probe. In this way the possibility of coherent transfer could be demonstrated.

The goal of this paper is to study this opportunity. In fact, we have developed a model representing the atomic sample as a simplified $\Lambda$-type level scheme consisting of an excited level and two pairs of ground state levels. Atoms are excited by a pulsed pump and by a cw probe laser: when the frequency of pumping laser is modulated and synchronized with the Larmor precession, the optical pumping efficiency resonantly enhances [Acosta(2006)] [Dehmelt(1957)] [Bell(1957)]. Because the two optical transitions share the same excited state, coherence transfer between the ground state levels is allowed. On the other hand coherence and population transfer is also possible by means of relaxation effects and it is interesting to investigate the importance of the latter on the predicted signal.

The paper is organized as follows. In Section 2 the model is widely discussed. In Section 3 we present and discuss the numerical results related to the optimization of the lasers parameters. Finally Section 4 contains the conclusions.

\section{Model}

Let us consider the simplified atomic model sketched in Fig. 1. 
|1)

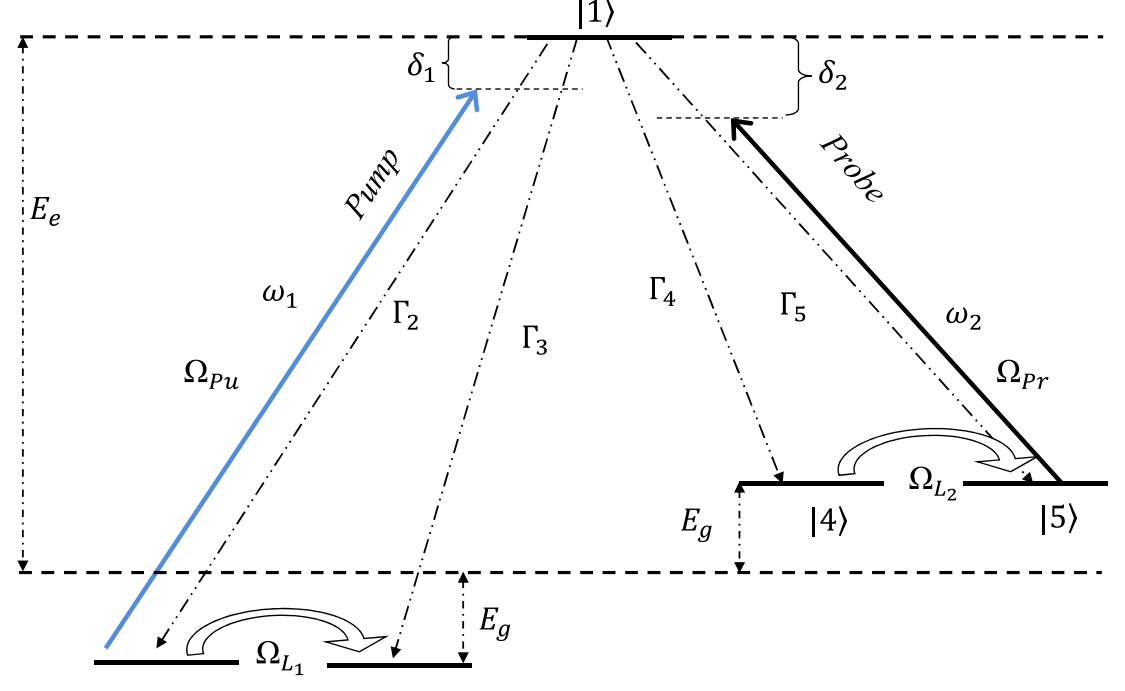

$|2\rangle$

|3〉

Figure 1: Schematic diagram of a five-level $\Lambda$-type atomic system, which consists of excited level $|1\rangle$ and two ground state levels with magnetic sublevels $|2\rangle,|3\rangle$ and $|4\rangle,|5\rangle$. The atomic system is driven by two laser fields, with $\delta_{1}$ being the pump laser detuning and $\delta_{2}$ the probe laser detuning. $\Gamma_{i}(i=2, \ldots, 5)$ is the spectral width of $|1\rangle \rightarrow|i\rangle$ transition.

It consists of one excited level $|1\rangle$ of energy $E_{e}$ and two doubly degenerate ground state levels of energy $-E_{g}(|2\rangle,|3\rangle)$ and $+E_{g}(|4\rangle,|5\rangle)$. To be more definite we can think of levels $(|2\rangle,|3\rangle)$ belonging to the $F=3$ ground hyperfine multiplet of ${ }^{133} \mathrm{Cs}$ atoms. More precisely $|2\rangle=\left|F=3, M_{F}=2\right\rangle,|3\rangle=\left|F=3, M_{F}=3\right\rangle$. Similarly $|4\rangle=\left|F=4, M_{F}=-4\right\rangle,|5\rangle=\left|F=4, M_{F}=-3\right\rangle$. The pump beam, of frequency $\omega_{1}$, is tuned to the transition $|2\rangle \rightarrow|1\rangle$ with variable detuning $\delta_{1}(t)$; the probe beam, of frequency $\omega_{2}$, is tuned to the transition $|5\rangle \rightarrow|1\rangle$ with detuning $\delta_{2}$. The Rabi frequencies are respectively $\Omega_{P r}$ and $\Omega_{P u}$. $\Omega_{L_{i}}$ are the Larmor frequencies of the $|2\rangle \rightarrow|3\rangle(i=1)$ and $|4\rangle \rightarrow|5\rangle(i=2)$ transitions. We assume that the spectral widths of the lasers are narrower than the atomic spectral linewidth. The pump laser frequency is modulated with a rectangular function signal whose period $T$ can be adjusted with respect to the Larmor one

$$
T=\kappa \frac{2 \pi}{\Omega_{L_{1}}} .
$$

The time dependence of pump detuning is shown in Fig. 2. The frequency modulation of the pump laser produces synchronous Zeeman optical pumping and a macroscopic magnetization build-up. If the modulation frequency is not synchronized with the Larmor frequency, the magnetizations associated with subsequent light pulses are randomly oriented and average to zero in few pulses (as shown in Fig. 8). On the other hand the same behavior is observed when the duty cycle of the modulating square wave is large, enforcing the idea that the pumping should not significantly disturb the 


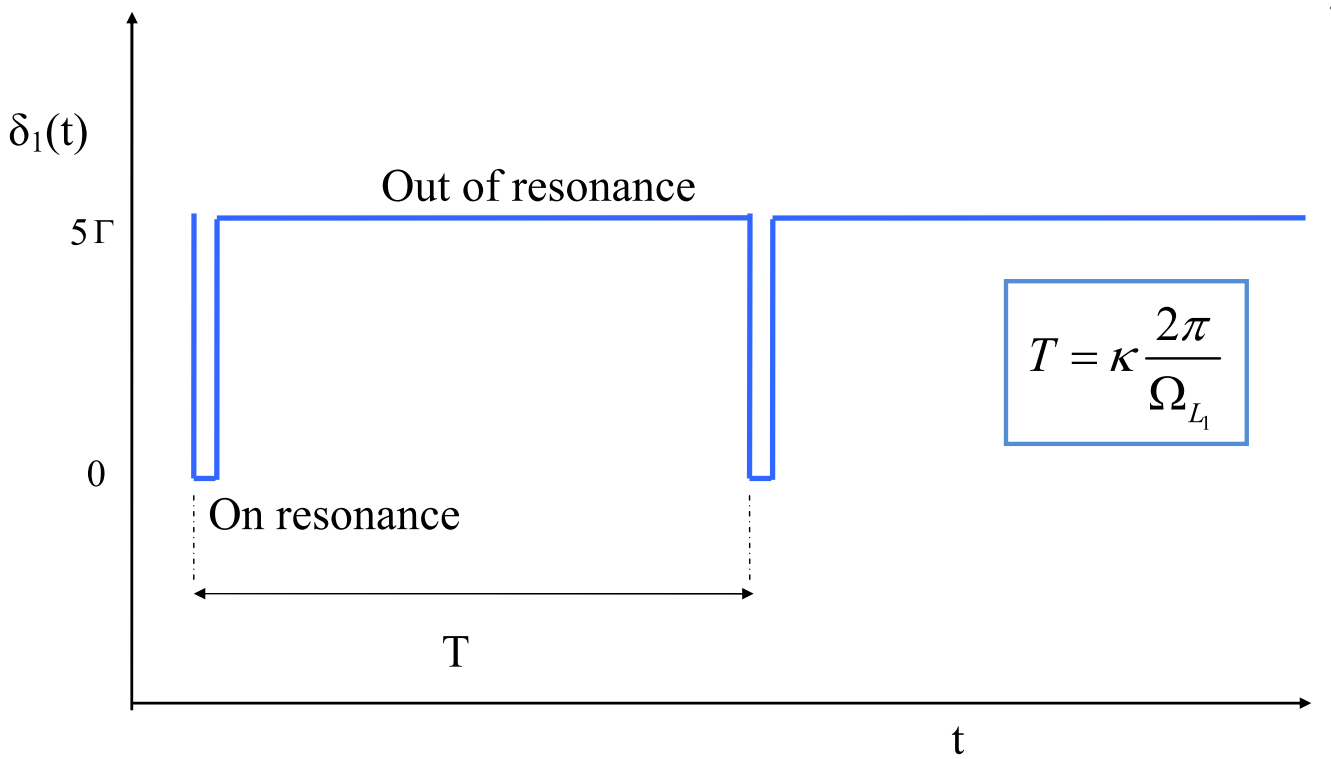

Figure 2: Typical rectangular signal function used to modulate the pump laser frequency. In the calculations a duty-cycle of $\sim 2 \%$ is assumed.

spins during the Larmor precession.

\subsection{Hamiltonian}

The total Hamiltonian is equal to the sum of the unperturbed term $H_{0}$ and the atomfield interaction addendum $H_{1}(t)$,

$$
H=H_{0}+H_{1}(t)
$$

In the basis of the $|j\rangle(j=1, \ldots, 5)$ states, the Hamiltonian $H_{0}$ reads (see Fig. 1$)$

$$
H_{0}=\left(\begin{array}{ccccc}
E_{e} & 0 & 0 & 0 & 0 \\
0 & -E_{g} & 0 & 0 & 0 \\
0 & 0 & -E_{g} & 0 & 0 \\
0 & 0 & 0 & E_{g} & 0 \\
0 & 0 & 0 & 0 & E_{g}
\end{array}\right)
$$

The atom-field interaction Hamiltonian $H_{1}(t)$ consists of a sum of the probe laser-atom interaction term $H_{P r}(t)$, the pump laser-atom interaction term $H_{P u}(t)$, and the magnetic field-atom interaction term $H_{B}(t)$ :

$$
H_{1}(t)=H_{P r}(t)+H_{P u}(t)+H_{B},
$$

where

$$
H_{B}=\hbar \frac{\Omega_{L_{1}}}{2}|2\rangle\left\langle 3\left|+\hbar \frac{\Omega_{L_{2}}}{2}\right| 4\right\rangle\langle 5|+H . c .,
$$


and in the rotating wave approximation the coupling to the optical fields reads

$$
\begin{aligned}
& H_{P u}(t)=\hbar \Omega_{P u} e^{-i\left(\omega_{1} t+\Phi(t)\right)}|1\rangle\langle 2|+H . c ., \\
& H_{P r}(t)=\hbar \Omega_{P r} e^{-i \omega_{2} t}|1\rangle\langle 5|+H . c .
\end{aligned}
$$

Here $\omega_{1}+\dot{\Phi}(t)$ is the instantaneous frequency of the pump laser (see Fig. 2). Transforming to the rotating frame specified by the unitary operator

$$
U(t)=|1\rangle\langle 1|+(|2\rangle\langle 2|+| 3\rangle\langle 3|) \mathrm{e}^{\left(i \omega_{1} t+\Phi(t)\right)}+(|4\rangle\langle 4|+| 5\rangle\langle 5|) \mathrm{e}^{i \omega_{2} t},
$$

we get the following expression for the transformed Hamiltonian $\widetilde{H}=U^{\dagger} H U+i U^{\dagger} \dot{U}$ :

$$
\widetilde{H}=\hbar\left(\begin{array}{ccccc}
0 & \Omega_{P u} & 0 & 0 & \Omega_{P r} \\
\Omega_{P u} & \delta_{1}(t) & \frac{\Omega_{L_{1}}}{2} & 0 & 0 \\
0 & \frac{\Omega_{L_{1}}}{2} & \delta_{1}(t) & 0 & 0 \\
0 & 0 & 0 & \delta_{2} & \frac{\Omega_{L_{2}}}{2} \\
\Omega_{P r} & 0 & 0 & \frac{\Omega_{L_{2}}}{2} & \delta_{2}
\end{array}\right)
$$

where (assuming $\hbar \equiv 1$ from now on)

$$
\begin{aligned}
& \delta_{1}(t)=\left(E_{e}+E_{g}\right)-\left(\omega_{1}+\dot{\Phi}(t)\right) \\
& \delta_{2}=\left(E_{e}-E_{g}\right)-\omega_{2}
\end{aligned}
$$

\subsection{Relaxation}

Proper description of relaxation processes in atomic systems requires the density matrix formalism, whose evolution is governed by optical Bloch equations:

$$
\frac{\partial \rho(t)}{\partial t}=-i[\widetilde{H}, \rho(t)]+\mathcal{L}_{D} \rho(t)=\mathcal{L}_{H} \rho(t)+\mathcal{L}_{D} \rho(t)
$$

where $\mathcal{L}_{D}$ represents the relaxation processes, which are taken into account in the following standard form:

$$
\left(\mathcal{L}_{D}\right)_{i j, k m}=-\delta_{i k} \delta_{j m} \Gamma_{i j}\left(1-\delta_{i j}\right)+\delta_{i j} \delta_{k m}\left(\Gamma_{k \rightarrow i}-\sum_{n} \Gamma_{i \rightarrow n} \delta_{k i}\right) .
$$

Here $\Gamma_{i \rightarrow j}$ is population transition rate from level $i$ to level $j$ while $\Gamma_{i j}=\Gamma_{j i}$ are the coherence damping parameters. The rates $\Gamma_{i \rightarrow j}$ describes spontaneous decay from the excited state to the ground states, population transfer in the ground states both between hyperfine levels with rates $\gamma_{24}^{L}, \gamma_{25}^{L}, \gamma_{34}^{L}, \gamma_{34}^{L}$ and between the Zeeman sublevels with rates $\gamma_{23}^{L} \gamma_{45}^{L}$. So we model the population transfer as follow

$$
\Gamma_{i \rightarrow j}=\left(\begin{array}{ccccc}
0 & \Gamma / 4 & \Gamma / 4 & \Gamma / 4 & \Gamma / 4 \\
0 & 0 & \gamma_{23}^{L} & \gamma_{24}^{L} & \gamma_{25}^{L} \\
0 & \gamma_{23}^{L} & 0 & \gamma_{34}^{L} & \gamma_{35}^{L} \\
0 & \gamma_{24}^{L} & \gamma_{34}^{L} & 0 & \gamma_{45}^{L} \\
0 & \gamma_{25}^{L} & \gamma_{35}^{L} & \gamma_{45}^{L} & 0
\end{array}\right)
$$

where we assumed equal branching ratio for the spontaneous emission from the excited state, which has the natural linewidth equal to $\Gamma$. The $\Gamma_{i j}$ matrix contains the 
damping due to the population transfer via the rates $G_{i}=\sum_{j} \Gamma_{i \rightarrow j}$, as well as a "phenomenological" part $\Gamma_{i j}^{p h e n}$ which describes dephasing processes unable to change the populations

$$
\Gamma_{i j}^{\text {phen }}=\left(\begin{array}{ccccc}
0 & 0 & 0 & 0 & 0 \\
0 & 0 & \gamma_{23}^{C} & \gamma_{24}^{C} & \gamma_{25}^{C} \\
0 & \gamma_{23}^{C} & 0 & \gamma_{34}^{C} & \gamma_{35}^{C} \\
0 & \gamma_{24}^{C} & \gamma_{34}^{C} & 0 & \gamma_{45}^{C} \\
0 & \gamma_{25}^{C} & \gamma_{35}^{C} & \gamma_{45}^{C} & 0
\end{array}\right)
$$

The total matrix is

$$
\Gamma_{i j}=\frac{G_{i}+G_{j}}{2}+\Gamma_{i j}^{p h e n} .
$$

Here $\gamma_{24}^{C}, \gamma_{25}^{C}, \gamma_{34}^{C}, \gamma_{34}^{C}$ and $\gamma_{23}^{C}, \gamma_{45}^{C}$ are the coherence relaxation rates between hyperfine and Zeeman levels respectively.

Various physical mechanisms affect the hyperfine and Zeeman relaxation rates. The most important contributions are related to collisions with the cell walls, with the buffer gas atoms and with atoms of the same kind giving rise to spin-exchange. A wide and detailed account can be found in [Vanier(1989)]. Here we are interested in those rates as phenomenological parameters only and we do not pursue further any microscopic identification. Moreover we do not take into account the Doppler broadening because the presence of buffer gas changes the atomic motion from balistic to diffusive (this is the main reason of using the buffer gas).

The density matrix time evolution was performed numerically using standard tools to solve initial value problems. We assumed that at $t=0$ the atoms start to interact with the external fields. After a transient which is related to the initial state $\rho(0)$, the system settles in the time-dependent stationary state. We have carefully checked that the stationary state is unrelated to $\rho(0)$ (as expected on general ground); additionally we found that the final state is reached faster starting the simulation with all the atoms in the excited level.

\section{Results}

We decided to focus our attention on the imaginary part of $\rho_{51}$, information which is directly comparable with the absorption of the probe beam. A typical time evolution of $\Im\left[\rho_{51}(t)\right]$ is shown in Fig. 3 .

At early time intervals $\Im\left[\rho_{51}(t)\right]$ shows a transient time dependence, then after many cycles of pump laser but very rapidly on the laboratory time scale, it reaches the stationary regime, which is of interest for our study. Vertical lines in Fig. 3 correspond to beginning of time intervals where the pump laser frequency fits the resonance (see Fig. 2). The absorption then oscillates at approximately the Rabi frequency. When the pump laser goes out of resonance the absorption get driven by the "free evolution" governed by the magnetic field. 


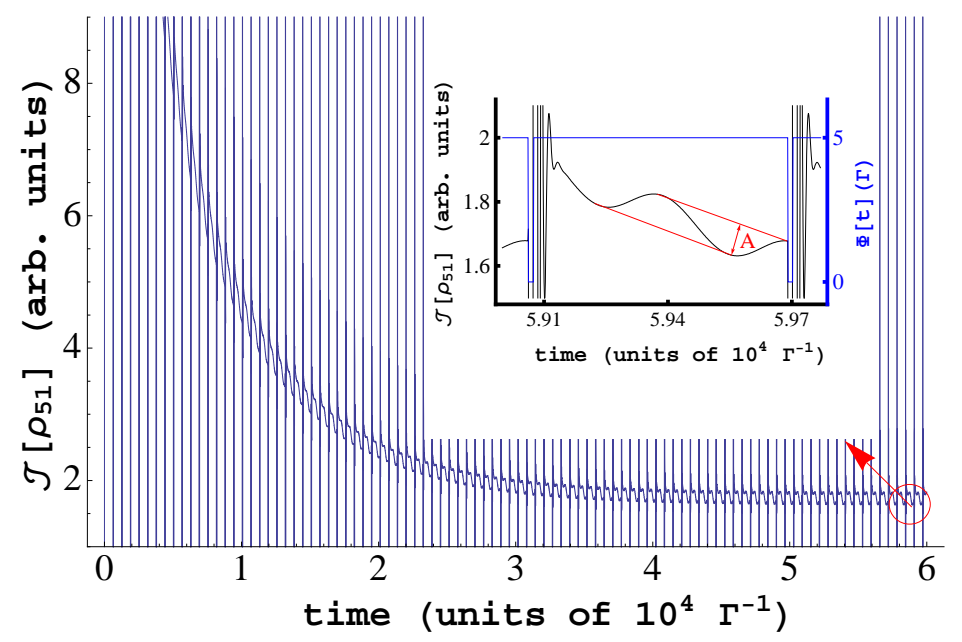

Figure 3: Time dependence of $\Im\left[\rho_{51}(t)\right]$. Inset shows the steady state dependence, where $A$ is the oscillation amplitude.

In other words one can think that, when the pump laser is out of resonance, the probe laser moves atoms from level $|5\rangle$ to level $|1\rangle$ so that the levels $|2\rangle,|3\rangle$ are populated by spontaneous emission from level $|1\rangle$ while level $|5\rangle$ (and accordingly $|4\rangle$ ) are depopulated. The process is inverted turning on the pump laser thus repopulating levels $|4\rangle$ and $|5\rangle$ via spontaneous decay. However a proper quantitative analysis is more complicated than this simple picture because of the incoherent population transfer between the ground states. We are interested in studying the behaviour of the Larmor's oscillation amplitude $A$ (see inset in Fig. 3) as a function of the various model parameters. Our numerical findings are reported in the following subsections.

\subsection{Time evolution of $\Im \rho_{51}(t)$ for different Larmor frequencies}

Time dependence of $\Im\left[\rho_{51}(t)\right]$ for different values of Larmor frequency (i.e. for the different values of external magnetic field) is shown in Fig. 4. Here assuming that $\Omega_{L_{1}}= \pm \Omega_{L_{2}}$ we specifically think that the ground levels refer to the ground hyperfine states of alkali atoms.

The $\Im\left[\rho_{51}(t)\right]$ amplitude $A$ takes a higher value for the case of $\Omega_{L}=0.025 \Gamma$ as compared with $\Omega_{L}=0.01 \Gamma$.

\subsection{Dependence of $\Im\left[\rho_{51}(t)\right]$ amplitude on Rabi frequency of pump laser}

As shown in Fig. 5, for small $\Omega_{P u}$ the amplitude of $\Im\left[\rho_{51}(t)\right]$ approaches zero, then increases and eventually saturates. In fact the more the probe laser is resonant the better the process is enhanced. Secondly at higher values of $\Omega_{P u}$, the atoms are "repumped" in $|4\rangle$ and $|5\rangle$ efficiently from $|2\rangle$ and $|3\rangle$.

In both circumstance the amplitude of absorption coefficient grows up, arriving at its saturation value. 


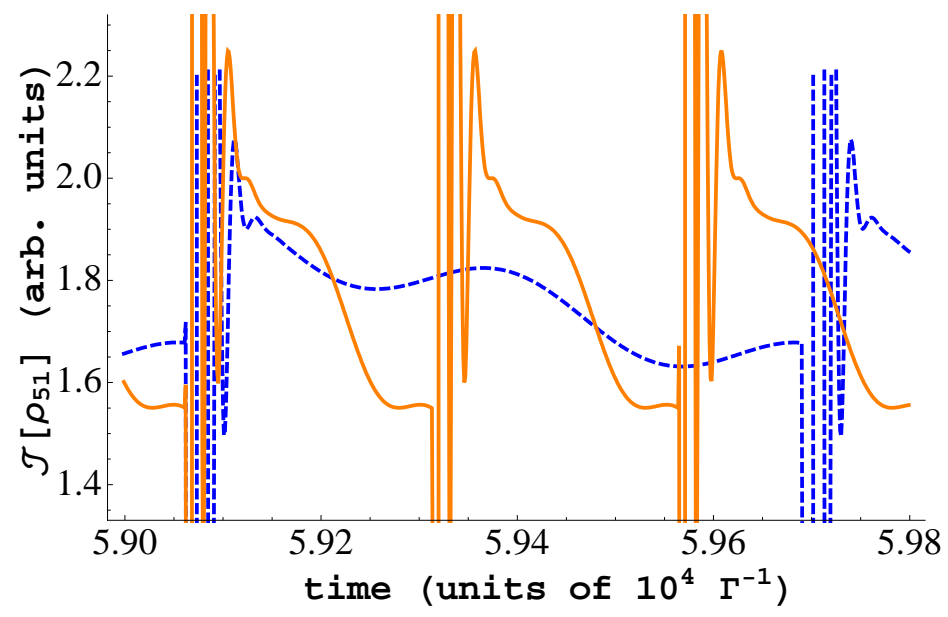

Figure 4: Time dependence of $\Im\left[\rho_{51}(t)\right]$ for $\Omega_{P u}=0.08 \Gamma, \Omega_{P r}=0.3 \Gamma, \Omega_{L}=0.01 \Gamma$ (dashed line), $\Omega_{L}=0.025 \Gamma$ (solid line).

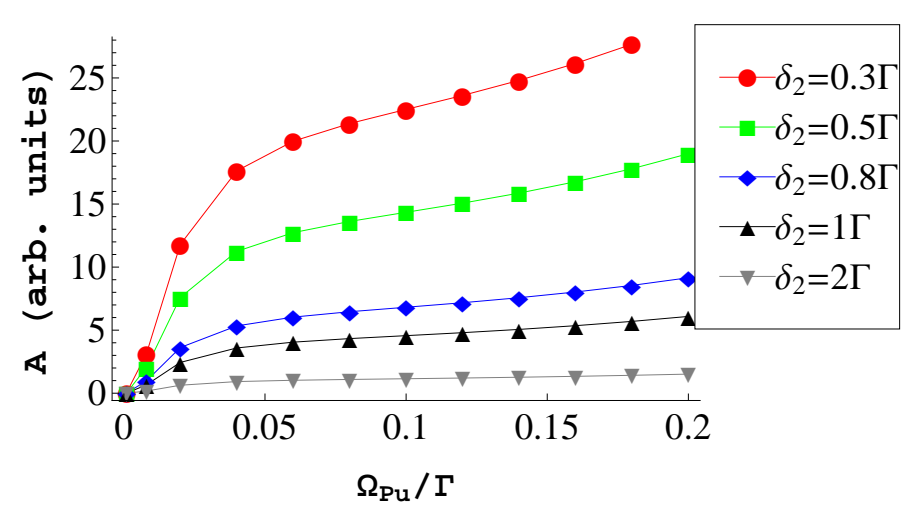

Figure 5: Dependence of $\Im \rho_{51}(t)$ amplitude on Rabi frequency of pump laser for different frequency detunings of probe laser rom the resonance $\left(\delta_{2}=0.3 \Gamma, \ldots, 2 \Gamma\right)$.

It is possible to have an roughly estimate of the saturation intensity of the pump laser using the textbook[Foot(2002)] formula

$$
I_{\text {sat }}=\frac{\pi}{3} \frac{h c}{\lambda^{3} \tau}
$$

which is exactly valid for a two level system. In our case for the resonance transition of Cesium ( $\lambda=852 \mathrm{~nm}$, lifetime $\tau=\Gamma^{-1}=30 \mathrm{~ns}$ ) the saturation intensity is $I_{\text {sat }}=1.3$ $\mathrm{mW}$ corresponding to $\Omega_{P u}=0.1 \Gamma$, which is in good agreement with the behaviour shown in Fig. 5

\subsection{Dependence of $\Im \rho_{51}(t)$ amplitude on Rabi frequency of probe laser}

Dependence of $\Im\left[\rho_{51}(t)\right]$ amplitude on Rabi frequency of probe laser for different Rabi frequencies of pump laser $\left(\Omega_{P u}=0.02 \Gamma, \ldots, 0.08 \Gamma\right)$ is shown in Fig. 6 . The plot corresponds to off-resonance $\left(\delta_{2}=1 \Gamma\right)$ frequency of the probe laser (Fig. 2). 


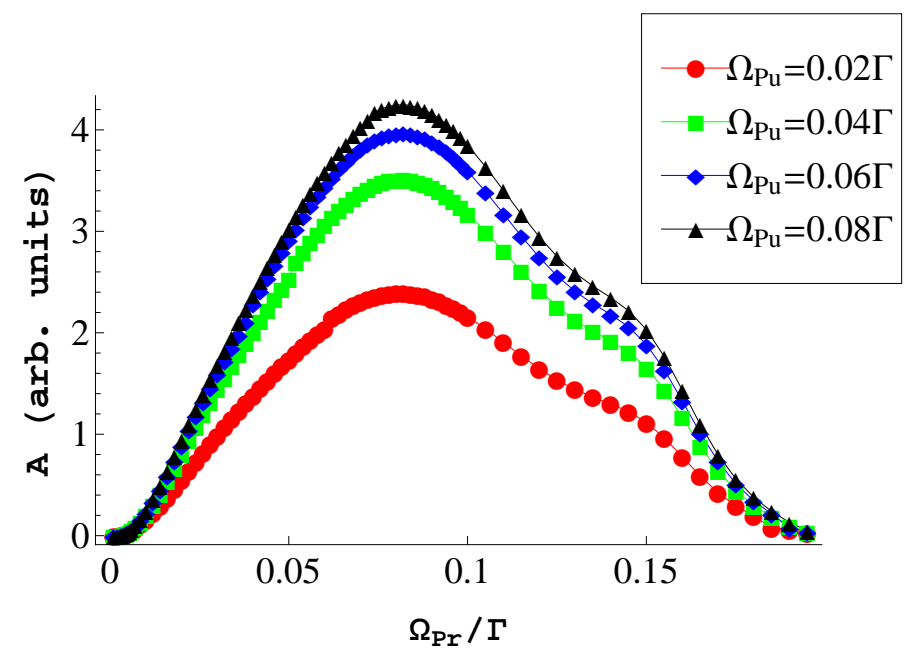

Figure 6: Dependence of $\Im\left[\rho_{51}(t)\right]$ amplitude $A$ on probe laser intensity for different values of pump laser Rabi frequency.

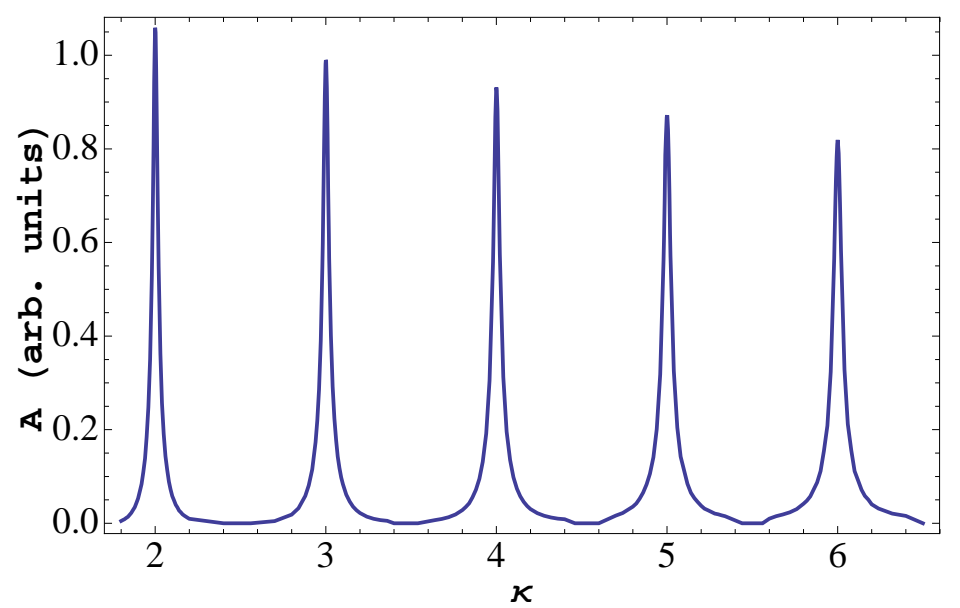

Figure 7: $\Im\left[\rho_{51}(t)\right]$ amplitude versus the modulation period of pump laser.

When the Rabi frequency of probe laser is zero, the amplitude of $\Im\left[\rho_{51}(t)\right]$ is zero too, and all atoms are moved to $|4\rangle$ and $|5\rangle$ levels by the pump laser action. With increasing of $\Omega_{P r}$, the amplitude $A$ increases too, reaching a maximum and then starts to decrease. The results can be explained thinking that the probe feels the precessing magnetization $\mathbf{M}_{2}$ related to the $|4\rangle$ and $|5\rangle$ levels, (namely $M_{2 x} \propto \rho_{45}+\rho_{54}$, $\left.M_{2 y} \propto i\left(\rho_{45}-\rho_{54}\right), M_{2 z} \propto \rho_{44}-\rho_{55}\right)$. Thus for small $\Omega_{P r}$ the signal increases as expected reaching a maximum. However when $\Omega_{P r}$ increases, the probe is more and more effective in shifting the atoms to levels $|2\rangle$ and $|3\rangle$, thus reducing the amplitude of $\mathbf{M}_{2}$ and eventually destroying it. 


\subsection{Dependence of $\Im\left[\rho_{51}(t)\right]$ amplitude on modulation period of the pump laser}

In Fig. 7 the behaviour of $A$ with respect to $\kappa$ is shown. The value $\kappa=1$ is missing from the picture because it was impossible to locate in a precise way the maximum and minimum positions of $\Im\left[\rho_{51}(t)\right]$. In fact in such a case the Larmor oscillation starts together with the Rabi oscillation (see Fig. 3) leading to unreliable results for $A$. For this reason we started the picture from $\kappa=2$ value.

At $\kappa=2$ i.e., when the pump laser is modulated with a frequency equal to twice Larmor frequency, $A$ takes the maximum value. In this case the frequency of pump laser is tuned to resonance exactly at the moment when the atomic spin completes one period of damped precession around the external magnetic field. When the frequency of pump laser is switched on to $|2\rangle \rightarrow|1\rangle$ with $\kappa$ not close at integer values, the $\Im\left[\rho_{51}(t)\right]$ amplitude decreases to zero, since the coherence in the ground levels gets destroyed and the asynchronous pumping is not able to restore the magnetization. The amplitude increases again when $\kappa$ approaches integers restoring the picture of synchronous pumping.

The above line of reasoning is confirmed comparing the time dependence of $\Im\left[\rho_{51}(t)\right]$ for $\kappa=1.6$ and $\kappa=2$ as shown in Fig. 8 .

\subsection{Dependence of $\Im\left[\rho_{51}(t)\right]$ on relaxation rates}

In this section we will investigate the dependence of $\Im\left[\rho_{51}(t)\right]$ amplitude on the longitudinal and coherence relaxation rates of the hyperfine and the Zeeman levels. This study is done at the optimum conditions for obtaining the maximum value of absorption amplitude, namely, $\Omega_{P u}=0.08 \Gamma, \Omega_{P r}=0.1 \Gamma, \delta_{2}=1 \Gamma$.

In Fig. 9 we present the dependence of $A$ on population relaxation rates, for the case of off-resonance probe laser detuning $\left(\delta_{2}=1 \Gamma\right)$ and the following choice of the relaxation parameters

$$
\begin{aligned}
& \gamma_{24}^{L}=2 \gamma_{25}^{L}=0.1 \gamma_{23}^{L}, \\
& \gamma_{34}^{L}=4 / 3 \gamma_{35}^{L}=0.3 \gamma_{23}^{L}, \\
& \gamma_{45}^{L}=\gamma_{23}^{L},
\end{aligned}
$$

which "advantages" the state $|4\rangle$ with respect to the state $|5\rangle$. However we have found that different, but comparable, choices of these parameters give qualitatively comparable results.

As is seen from the upper part of Fig. 9, $A$, for small values of $\Omega_{P r}$, does not vanish when the relaxation is zero, since in this case the atoms are moved by pump laser from level $|2\rangle$ to level $|1\rangle$ followed by spontaneous decay to $|5\rangle$ and $|4\rangle$. As a result, one can see the almost undisturbed Larmor oscillation of $\Im \rho_{51}(t)$ on $|5\rangle \rightarrow|1\rangle$ transition. When the longitudinal relaxation rate increases, the amplitude rapidly decreases indicating that the relaxation processes are very effective in destroying the atomic magnetization. 


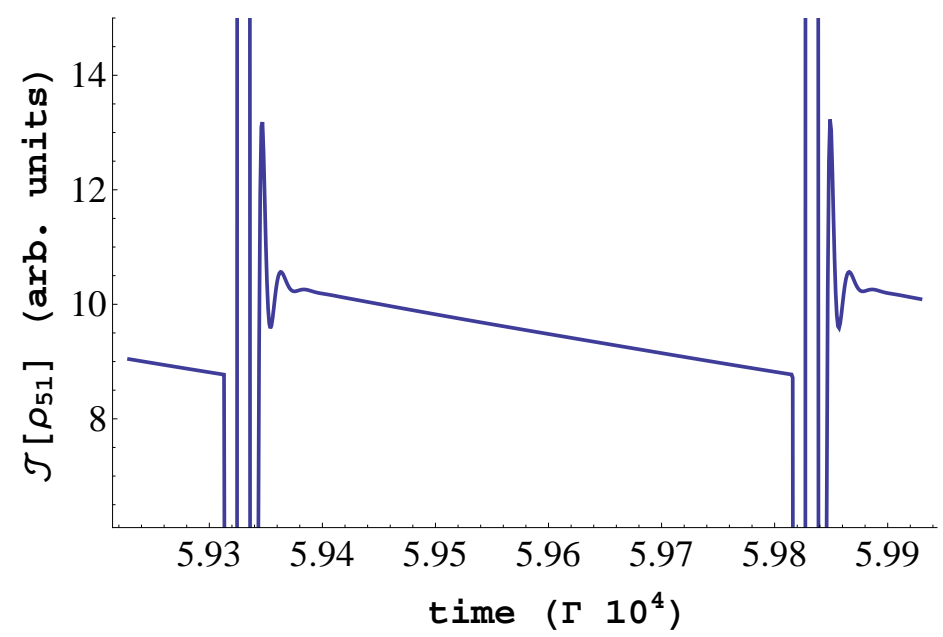

(a) $\kappa=1.6$

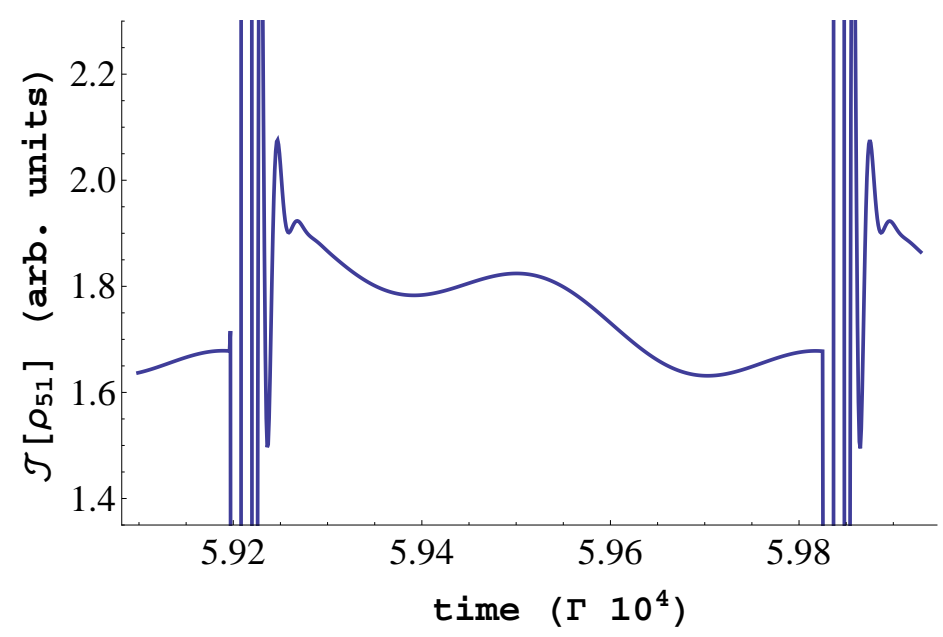

(b) $\kappa=2$

Figure 8: $\Im \rho_{51}(t)$ dependence on time when $\Omega_{P u}=0.08 \Gamma, \Omega_{P r}=0.3 \Gamma$.

On the other hand, for higher values of $\Omega_{P r}$ and zero relaxation, the atoms are continuously "pumped off" thus depleting the levels $|4\rangle$ and $|5\rangle$. Accordingly the Larmor oscillations vanish. Increasing the relaxation the amplitude $A$ increases reaching a maximum and then decreases. It seems that the parameter $\gamma_{23}^{L}$ drives a twofold dynamics. For small values the relaxation contrasts the pumping effect of $\Omega_{P r}$, in fact the higher the value of $\gamma_{23}^{L}$ the more the atoms that incoherently "arrive" in the ground states $|4\rangle$ and $|5\rangle$, ready to be pumped out by the probe laser. This regime keeps on until $\gamma_{23}^{L}$ reaches a value where the dephasing effects become overwhelming and every magnetization is gradually destroyed, giving a vanishing amplitude $A$. In Fig. 10 is shown the behavior of $A$ when the Zeeman population relaxation is turned off.

From the picture inspection it seems that incoherent population exchange between the hyperfine multiplets is needed to observe appreciable signals. In fact when $\gamma_{L}$ is zero no Larmor oscillations are predicted. The explanation is as before: the probe 

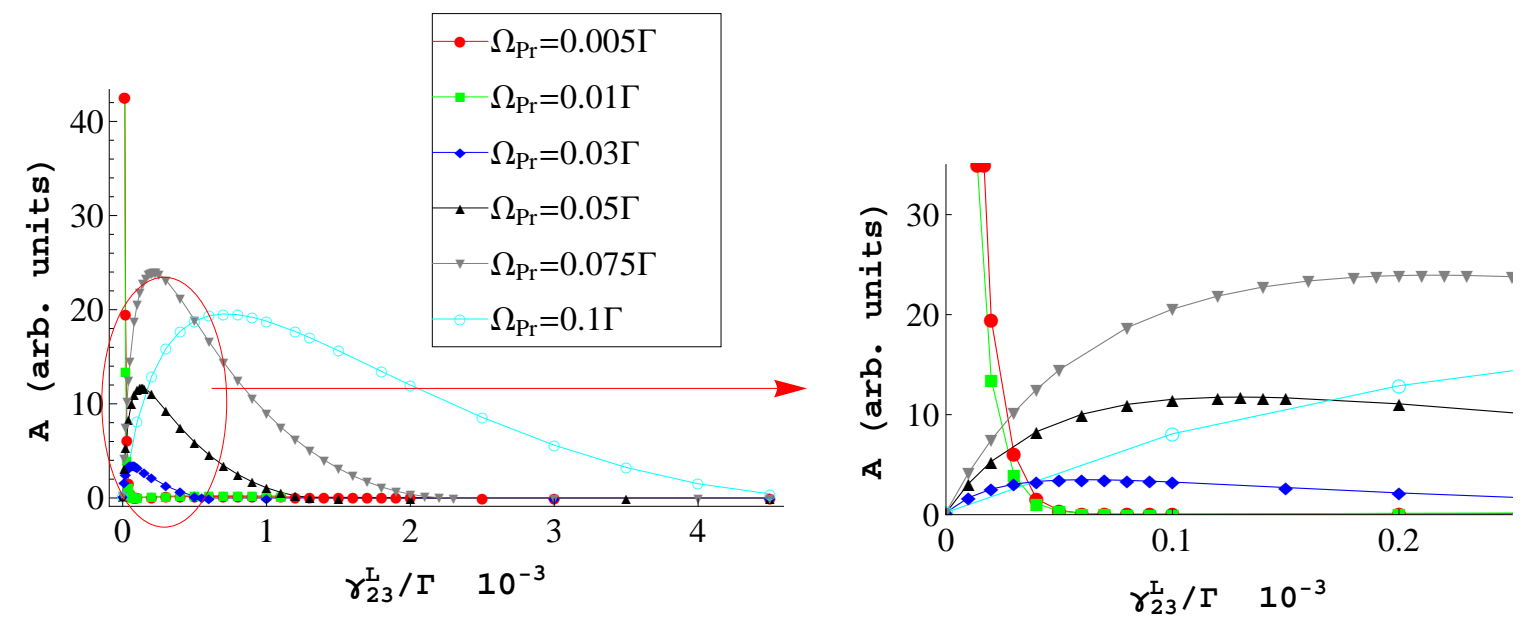

Figure 9: Amplitude of absorption coefficient on $|5\rangle \rightarrow|1\rangle$ transition versus $\gamma_{2,3}^{L}$ with the choice of the other $\gamma$ outlined in 17 and for different values of $\Omega_{P r}$. The cases with $\left(\Omega_{P r}=0.005 \Gamma, 0.01 \Gamma, 0.03 \Gamma, 0.05 \Gamma, 0.075 \Gamma, 0.1 \Gamma\right)$ are magnified 10 times for clarity. In the upper part is shown the initial region, while in the lower part the full picture is displayed.

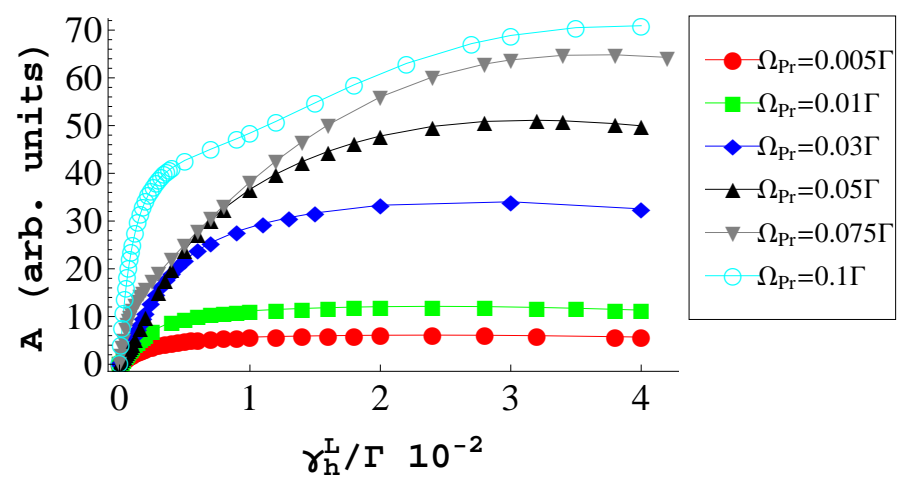

Figure 10: Dependence of $A$ from "hyperfine" relaxation rate for different values of the probe laser Rabi frequency as shown in the inset. The other relevant parameters are $\Omega_{P u}=0.08 \Gamma, \delta_{2}=1 \Gamma, \gamma_{24}^{L}=0.2 \gamma_{L}, \gamma_{25}^{L}=0.1 \gamma_{L}, \gamma_{34}^{L}=0.4 \gamma_{L}, \gamma_{35}^{L}=0.1 \gamma_{L}$, while $\gamma_{23}^{L}=\gamma_{45}^{L}=0$. As before, different but comparable choices of these parameters give the same qualitative results. The Larmor frequencies are $\Omega_{L_{1}}=\Omega_{L_{2}}=10^{-2} \Gamma$.

laser, in the stationary state, depletes in a very efficient way the states $|4\rangle$ and $|5\rangle$. On the contrary with relaxation, some population is supplied to the $|4\rangle$ and $|5\rangle$ states and then probed by the laser. Interestingly enough, the calculated amplitude shows a saturation effect with increasing relaxation, demonstrating that the Zeeman relaxation is responsible of the signal destruction (see Fig. 9). This conclusion is confirmed also by Fig. 11 where the only non-zero parameters are $\gamma_{2,3}^{L}=\gamma_{4,5}^{L}$. 


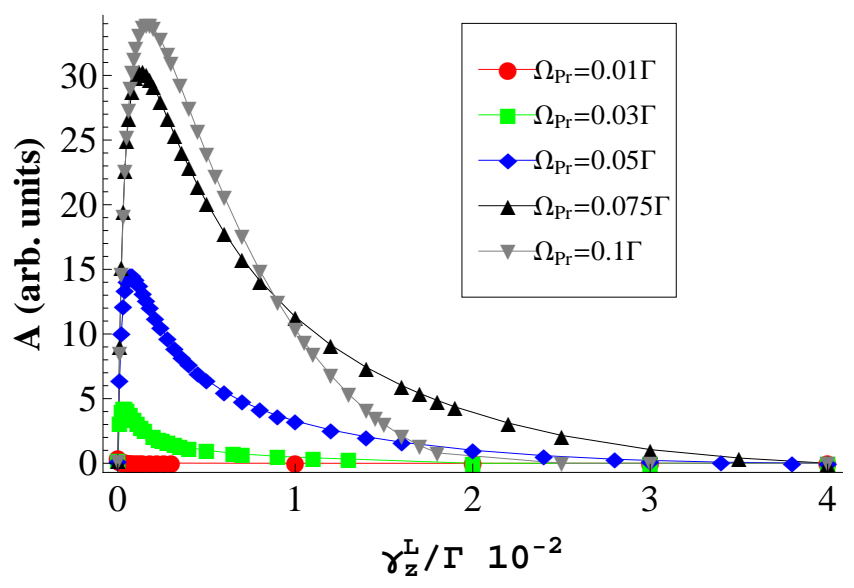

Figure 11: Behavior of $A$ with respect to the Zeeman population relaxation. The lasers parameters and Larmor frequencies are as before. The only non-zero relaxation parameters are $\gamma_{2,3}^{L}=\gamma_{4,5}^{L}$.

\section{Conclusions}

We have investigated the interaction of a $\Lambda$-type five-level atomic system (atomic vapor in a buffered cell) with two, pump and probe, laser beams, in the presence of an external, time independent magnetic field. The pump laser frequency is modulated in time by a rectangular function synchronized with the Larmor precession. The probe laser absorption shows the Larmor oscillation too and we characterized the amplitude of these oscillations with respect to the model parameters.

We found that the system behaves as expected with regard to the lasers parameters, but an interesting effect is put in evidence when one considers the influence of the relaxation. In fact we have shown that an amount of relaxation, in some circumstances, it is needed in order to keep the signal at good level. Concerning the application of this system as a magnetometer, the sensitivity limit as in other atomic magnetometers is set by the contrast of the observed spectral features, being the signal proportional to the narrow peak amplitude and the noise (assuming a shot noise limited condition) proportional to the background, this value would depend on the specific values of the relaxation rates. As an example, the contrast can be visualized in the inset of Fig 3, as the ratio between $A$ and the average steady state value of $\Im\left[\rho_{51}(t)\right]$. Summarizing we have shown that an incoherent population transfer in atomic levels plays an important role.

\section{Acknowledgments}

The authors are grateful to Y.Dancheva and V.Biancalana from the Magnetometer group of Department of Physics of Siena University for numerous discussions and suggestions and a critical reading of the manuscript. The authors would like to thank D.Sarkisyan, 
Yu.Malakyan and A.Kirakosyan for valuable discussions.

\section{References}

[Kastler(1950)] A. Kastler Some suggestions concerning the production and detection by optical means of inequalities in the populations of levels of spatial quantization in atoms. Application to the Stern and Gerlach and magnetic resonance experiments J. Phys Radium 11,255 (1950).

[Happer(1972)] W. Happer, Optical Pumping Rev. Mod. Phys. 44, 169-250, (1972).

[Series (1966)] G. W. Series, Theory of the modulation of light in optical pumping experiments Proc. Phys. Soc. 88, 957-968 (1966).

[Shah(2010)] V. Shah, G. Vasilakis, and,M. V. Romalis, High bandwidth atomic magnetometery with continuous quantum nondemolition measurements Phys. Rev. Lett. 104,013601 (2010).

[Schwindt(2004)] P. D. D. Kastler, S. Knappe, V. Shah, L. Hollberg, J. Kitching, Li.-A. Liew, J. Moreland, Chip-scale atomic magnetomete Appl. Phys. Lett. 85,6409-6411 (2004).

[Ledbetter(2008)] M. P. Ledbetter, I. M. Savukov, D. Budker, V. Shah, S. Knappe, J. Kitching, D. J. Michalak, S. Xu, A. Pines, Zero-field remote detection of NMR with a microfabricated atomic magnetometer Proc. Natl. Acad. Sci. USA 105,2286-2290 (2008).

[Belfi(2009)] J. Belfi, G. Bevilacqua, V. Biancalana, S. Cartaleva, Y. Dancheva, K. Khanbekyan, and,L. Moi, Dual channel self-oscillating optical magnetometer Josa B 26 , 910916 (2009).

[Acosta(2006)] V. Acosta, M. P Ledbetter, S. M Rochester, and, D. Budker, Nonlinear magnetooptical rotation with frequency-modulated light in the geophysical field range Phys. Rev. A 73, 053404 (2006).

[Dehmelt(1957)] H. G. Dehmelt, Modulation of a light beam by precessing atoms Phys. Rev. 105, 1924-1925 (1957).

[Bell(1957)] W. Bell, A. L. Bloom, Optical detection of magnetic resonance in alkali metal vapor Phys. Rev. 107, 1559-1565 (1957).

[Foot(2002)] C. J. Foot Atomic Physics 142, (2005).

[Vanier(1989)] J. Vanier,C. Audoin The quantum physics of atomic frequency standards volume 1 $308,(1989)$. 